\title{
Author Correction: Transient Scute activation via a self-stimulatory loop directs enteroendocrine cell pair specification from self-renewing intestinal stem cells
}

Jun Chen, Na Xu, Chenhui Wang, Pin Huang, Huanwei Huang, Zhen Jin, Zhongsheng Yu, Tao Cai, Renjie Jiao and Rongwen $\mathrm{Xi}$

Correction to: Nature Cell Biology https://doi.org/10.1038/s41556-017-0020-0, published online 15 January 2018.

In the version of this Article originally published, the author had misnumbered the reference citations in the Methods, using numbers 1-14 instead of 46-59. These errors have now been corrected in all online versions of the Article.

Published online: 19 April 2018

https://doi.org/10.1038/s41556-018-0053-Z

\section{Author Correction: Segregation of mitochondrial DNA heteroplasmy through a developmental genetic bottleneck in human embryos}

\author{
Vasileios I. Floros, Angela Pyle, Sabine Dietmann, Wei Wei, Walfred W. C. Tang, Naoko Irie, Brendan Payne, \\ Antonio Capalbo, Laila Noli, Jonathan Coxhead, Gavin Hudson, Moira Crosier, Henrik Strahl, Yacoub Khalaf, \\ Mitinori Saitou, Dusko Ilic, M. Azim Surani and Patrick F. Chinnery (D)
}

Correction to: Nature Cell Biology https://doi.org/10.1038/s41556-017-0017-8, published online 15 January 2018.

In the version of this Letter originally published, an author error led to the affiliations for Brendan Payne, Jonathan Coxhead and Gavin Hudson being incorrect. The correct affiliations are: Brendan Payne: ${ }^{3}$ Wellcome Trust Centre for Mitochondrial Research, Institute of Genetic Medicine, Newcastle University, Newcastle upon Tyne, UK. 'Institute of Neuroscience, Newcastle University, Newcastle upon Tyne, UK; this is a new affiliation 6 and subsequent existing affiliations have been renumbered. Jonathan Coxhead: ${ }^{11}$ Genomic Core Facility, Institute of Genetic Medicine, Newcastle University, Newcastle upon Tyne, UK; this is a new affiliation 11 and subsequent existing affiliations have been renumbered. Gavin Hudson: ${ }^{3}$ Wellcome Trust Centre for Mitochondrial Research, Institute of Genetic Medicine, Newcastle University, Newcastle upon Tyne, UK. In addition, in Fig. 2d, the numbers on the $x$-axis of the left plot were incorrectly labelled as negative; they should have been positive. These errors have now been corrected in all online versions of the Letter.

Published online: 19 April 2018

https://doi.org/10.1038/s41556-018-0064-9 